\section{Nonformulary Medication Orders and Discharge Discrepancies}

We applaud Chang and others ${ }^{1}$ for their innovative study detailing the labour costs associated with nonformulary medication orders in an acute care hospital. The review of medications for inclusion on a hospital formulary typically includes a clinical or therapeutic evaluation, as well as a budget analysis. Historically, such budget analyses were based solely on the acquisition cost of the drug being reviewed. Chang and others ${ }^{1}$ showed that the cost of pharmacists' time required for dealing with nonformulary medication orders should also be considered. However, another important aspect of the formulary drug review process is the impact on the patient of changing a medication that is deemed (by hospital policies) to be nonformulary.

We have previously demonstrated that changing a patient's prior-to-admission proton pump inhibitor (PPI) to the hospital's formulary PPI may yield cost savings (through therapeutic substitution), but this practice can also lead to a significant increase in discharge medication discrepancies. ${ }^{2}$ When our hospital's formulary PPI was the same as the BC provincial formulary PPI, there was a $27 \%$ rate of discharge medication discrepancies for PPIs. After the hospital's formulary PPI was changed, and was no longer aligned with the $\mathrm{BC}$ provincial formulary PPI, the rate of discharge discrepancies for PPIs increased to $49 \%$ ! Thus, while it may have been beneficial from an economic standpoint to change a patient's PPI to the hospital formulary PPI, given therapeutic equivalence and cost savings, it had a negative impact on discharge medication discrepancies.

Use of automatic or therapeutic substitution is commonplace in many hospitals, to change a patient's prior-to-admission medication to the hospital formulary equivalent. The use of automatic substitution is the most efficient way to deal with nonformulary medication orders in terms of pharmacists' time. However, as we found, it can significantly increase the rate of medication discharge discrepancies.

This effect on patients' discharge prescriptions and discharge medication reconciliation is not given high priority in many hospital formulary drug reviews. Ideally, reviews of drugs for inclusion on hospital formularies should factor in therapeutic equivalence, drug acquisition cost, cost of pharmacy staff labour, and effects on the patient and patient safety. Hospital drug formularies and automatic substitutions serve administrative and budgetary goals well, but we should ensure that such practices do not contribute to medication discrepancies.

\section{References}

1. Chang E, Kinkade A, Tung ACH, Tejani AM. Going through the motions: a time-and-motion study of workload associated with nonformulary medication orders. Can J Hosp Pharm. 2017;70(2):133-7.

2. Chua D, Chu E, Lo A, Lo M, Pataky F, Tang L, et al. Effect of misalignment between hospital and provincial formularies on medication discrepancies at discharge: PPITS (Proton Pump Inhibitor Therapeutic Substitution) study. Can J Hosp Pharm. 2012;65(2):98-102.

Doson Chua, BSC(Pharm), PharmD, FCSHP, BCPS(AQ)

Clinical Pharmacy Specialist - Cardiology

Cindy San, BSc(Pharm), PharmD

Clinical Pharmacy Specialist - Emergency Medicine

St Paul's Hospital, Providence Health Care

Vancouver, British Columbia

Doson Chua is also a Clinical Professor with the Faculty of Pharmaceutical Sciences, The University of British Columbia, Vancouver, British Columbia.

Competing interests: None declared 